\title{
Linking up Pressure, Chemical Potential and Thermal Gradients
}

\author{
François Montel $^{1 \S}$, Hai Hoang ${ }^{2}$, Guillaume Galliero ${ }^{1}$ \\ ${ }^{1}$ Laboratoire des Fluides Complexes et leurs Réservoirs, UMR-5150 CNRS/TOTAL/Univ Pau \\ \& Pays Adour, E2S, 64000, Pau, France \\ ${ }^{2}$ Institute of Fundamental and Applied Sciences, Duy Tan University, 10C Tran Nhat Duat \\ Street, District 1, Ho Chi Minh City 700000, Viet Nam
}

§francois.montel@univ-pau.fr

\begin{abstract}
Petroleum reservoirs are remarkable illustrations of the impact of a thermal gradient on fluid pressure and composition. This topic has been extensively studied during the last decades to build tools that are required by reservoir engineers to populate their models. However, one can get only a very limited number of representative samples from a given reservoir and assessing connectivity between all sampling points is often a key issue. In some extreme cases, the whole reservoir fluid properties must be derived from a single point to define the field development plan. To do so, available models are usually not satisfactory as they need too many parameters and so cannot be considered as predictive tools. We propose in this work a comprehensive approach based on the irreversible thermodynamics principles to derive the relationships between pressure, chemical potentials and thermal gradients in porous media. It appears that there is no need for additional assumptions, it is just a matter of a making the right choices along theoretical developments. One of the most important steps is to express the full pressure gradient. As a final result, we obtain the chemical potential gradients for all components of the mixtures that can be easily translated in term of compositions through Equation of State modelling. The most important features of the final expressions are: (i) the species relative separation in a thermal field is sensitive to the relative diffusion coefficients at stationary state. In porous media, the separation is sensitive to the permeability when the overall mobility is similar to diffusive mobility; (ii) the magnitude of the separation depends on the residual entropy of the species; (iii) the separation is not simply balanced by the average residual entropy. The balance is modified by the relative diffusion mobility of the components; (iv) in low permeability porous media, the thermal gradient induces a pressure gradient proportional to the fluid residual entropy. As a validation, the proposed approach has been applied on a reservoir fluid subjected to a geothermal gradient and compared with non-equilibrium molecular dynamics simulation results at the stationary state.
\end{abstract}




\section{Introduction}

The objective of this paper is to provide a general framework for the evaluation of the impact of a thermal gradient on fluid pressure and composition, i.e. thermodiffusion and related phenomena, in a porous medium at the stationary state. The main driver for this work is the development of a generic macroscopic formalism to describe the fluid distribution in petroleum reservoirs before production but many other applications of the proposed approach could be found in natural and industrial processes, see for example the recent review by Köhler and Morozov [1]. In petroleum industry, this topic is discussed in detailed in many recent textbooks $[2,3]$, and correlations for the thermodiffusion parameters, despite their intrinsic limitations [1], are commonly used by reservoir engineer to populate their reservoir models.

In some field cases, the geothermal gradient induces compositional gradients of the same order of magnitude than the gravitation [4-5]. Since the gravitational segregation is well quantified, the current practice in the petroleum industry consists in tuning the thermodiffusion parameters in order to match the available compositional field data. However, it happens quite often that the fluid column species distribution must be established from a very limited number of samples, sometimes only one as it is the case when data are coming from the discovery well. And it is of primary importance to get indisputable estimation of the thermodiffusion parameters to assess the fluid column species distribution and consequently oil and gas accumulation. A typical critical situation is the case of a discovery well crossing an oil pool, down-dip a large accumulation, for which a crucial question arises: is there a gas cap in the reservoir or not and if any where is it located? The symmetrical critical situation is encountered when the discovery well crosses the gas phase only with a possible oil rim down-dip.

Thus, building a predictive model to describe fluid distribution in oil and gas reservoirs at the stationary state is a challenge that may be a game changer for the petroleum industry. Following a previous work on the subject [6], in which the conditions for application of the stationary state model was discussed, the proposed article is a step towards that direction. The article is organized as follows. In section II, the theoretical framework is provided. Details on the new predictive approach is described in section III. Then, the methodology is applied and validated on a practical case in section IV. Finally, the main outcomes of this work are summarized in section V.

\section{Theoretical framework}

To derive the conservation equations and the related flux-forces relations as proposed in this work, our main hypothesis are: 
a. the average fluid velocity is slow enough to allow linearization of the fluid transport equation and to neglect inertial terms,

b. the local equilibrium hypothesis is valid in the fluid phase,

c. the fluid phase is homogeneous,

d. the transport and conservation equations can be homogenized in a simple manner regarding thermodynamic variables and properties.

A comprehensive discussion on these assumptions when dealing with oil and gas reservoir fluids can be found in Ref. [6].

\subsection{Material balance equation}

To write the material balance equation per species, we consider an elementary representative volume of the porous medium filled with a reservoir fluid, in which $n_{i}$ is the number of moles of component " $i$ " in this elementary volume.

$$
\frac{\partial n_{i}}{\partial t}+\nabla \mathbf{J}_{\mathbf{i}}=\mathbf{0}
$$

where, $\mathbf{J}_{\mathbf{i}}$ is the total molar flux of each component, which is a linear function of the thermodynamic forces, composed of three contributions (advection, thermodiffusion and molar diffusion). It can be expressed in terms of molar quantities in the absence of an inertial term (hypothesis “a") [6,7]:

$$
\mathbf{J}_{\mathbf{i}}=-n_{i} L_{P}\left(\frac{\mathrm{V} \nabla P-M \mathbf{g}}{T}\right)+n_{i} L_{i q} \nabla\left(\frac{1}{T}\right)+n_{i} \sum_{j} L_{i j}\left(\frac{M_{j} \mathbf{g}}{T}+\nabla\left(-\frac{\mu_{j}}{T}\right)\right)
$$

where $P$ is the pressure, $T$ the temperature, $\mu_{i}$ is the molar contribution of component " $i$ " to the free energy of the fluid in the porous medium, it is the chemical potential in absence of specific interactions between the component " $i$ " and the solid phase [6], $g$ is the gravitational acceleration, $M$ is the molecular weight, $V$ is the molar volume of the fluid, and $L_{p}, L_{i q}, L_{i j}$ are phenomenological coefficients related to advection (i.e. barodiffusion), thermodiffusion and molar diffusion respectively.

From the formulation of the molar flux per species, it is possible to deduce the advective fluid velocity which is simply the sum of the advective contributions of all components:

$$
\mathbf{U}=-\frac{1}{\rho} \sum_{i} n_{i} M_{i} L_{P}\left(\frac{\mathrm{V} \nabla P-M \mathbf{g}}{T}\right)=-\frac{L_{p}}{T}(\nabla P-\rho \mathbf{g})
$$

where, $\rho$ is the density. The sum of the thermodiffusive and diffusive contributions is nil in a closed system. However, it should be kept in mind that, in many petroleum reservoirs, there 
exist some stationary light gas component fluxes coming from deep sources through water saturated surrounding rocks, i.e. open systems. In such situations, the sum of these contributions are not nil at the stationary state [6].

\subsection{Energy equation}

If we neglect the viscous dissipation, the energy conservation equation can be written as:

$$
\frac{\partial H_{m}}{\partial t}=\nabla \cdot \mathbf{J}_{\mathbf{T}}
$$

where $H_{m}$ is the enthalpy of the saturated porous medium and the total heat flux $\mathbf{J}_{\mathrm{T}}$ is expressed by:

$$
\mathbf{J}_{\mathbf{T}}=L_{q q} \nabla\left(\frac{1}{T}\right)-L_{P} H\left(\frac{\mathrm{V} \nabla P-M \mathbf{g}}{T}\right)+\sum_{j} L_{q j}\left(\frac{M_{j} \mathbf{g}}{T}+\nabla\left(-\frac{\mu_{j}}{T}\right)\right)
$$

in which ones recognizes a pure conduction contribution, an advective term and a thermodiffusion (Dufour) term with the corresponding phenomenological coefficients $L_{q q \text {, }}$ $L_{p}, L_{q j}$, respectively. $H$ is the enthalpy defined as, $H=\sum_{i} n_{i} h_{i}$, where $h_{i}$ is the partial molar enthalpy of component $i$.

The contribution of the molar flux of component " $i$ " to the heat flux can be written as:

$$
\mathbf{J}_{\mathbf{q i}}=-n_{i} h_{i} L_{P}\left(\frac{\mathrm{V} \nabla P-M \mathbf{g}}{T}\right)+n_{i} h_{i}^{*} \sum_{j} L_{i j}\left(\frac{M_{j} \mathbf{g}}{T}+\nabla\left(-\frac{\mu_{j}}{T}\right)\right)
$$

in which $h_{i}^{*}$ is the molar enthalpy of component $i$ carried out during molar diffusion. As for the difference between overall permeability and individual mobility [6], we distinguish between the enthalpy of the component in the bulk fluid flow $h_{i}$ and the enthalpy transported by the same component, in and out the elementary volume, through a diffusion process, $h_{i}^{*}$. This difference is crucial. A single molecule carries only its own enthalpy. The energy balance of a single molecule in and out the elementary volume is not equal to its partial enthalpy in the fluid phase multiplied by the temperature difference if it moves alone independently from other molecules. $h_{i}^{*}$ differs from the partial enthalpy of the component " $i$ ".

From the previous equations, i.e. Eqs. (5-6), it is straightforward to deduce that the total heat flux can be written as :

$$
\mathbf{J}_{\mathbf{T}}=L_{q q} \nabla\left(\frac{1}{T}\right)-L_{P} H \frac{\rho}{M}\left(\frac{\nabla P-\rho \mathbf{g}}{T}\right)+\sum_{i} \sum_{j} n_{i} L_{i j} h_{i}^{*}\left(\frac{M_{j} \mathbf{g}}{T}+\nabla\left(-\frac{\mu_{j}}{T}\right)\right)
$$

\subsection{Molar flux}


The reciprocal Onsager relations imply that the contribution of molar diffusive fluxes to the total heat flux must be consistent with the contribution of the thermal gradient to the molar diffusive fluxes. The coefficients are equal when the right thermodynamic forces and conjugate variables are selected [7]. Therefore, in the proposed formulation, the phenomenological thermodiffusion coefficients are:

$$
L_{i q}=L_{q i}=\frac{1}{n_{i}} \sum_{j} n_{j} L_{i j} h_{i}^{*}
$$

and the component fluxes, Eq. (2), can be expressed as :

$$
\mathbf{J}_{\mathbf{i}}=-n_{i} L_{P}\left(\frac{\mathrm{V} \nabla P-M \mathbf{g}}{T}\right)+\sum_{j} n_{j} L_{i j} h_{i}^{*} \nabla\left(\frac{1}{T}\right)+n_{i} \sum_{j} L_{i j}\left(\frac{M_{j} \mathbf{g}}{T}+\nabla\left(-\frac{\mu_{j}}{T}\right)\right)
$$

It should be noted that a similar approach was initially proposed by Haase [8] and applied to petroleum reservoir by Pedersen and Lindeloff in 2003 [9].

It is possible to go even further by using the fact that all the thermodynamic properties of a component $i$ can be derived from its chemical potential and introducing the chemical potential gradient at constant temperature and the molar entropy obtains:

$$
\frac{\nabla \mu_{i}}{T}=\frac{-s_{i} \nabla \mathrm{T}+\nabla_{T} \mu_{i}}{T}
$$

Finally, the driving force for molecular diffusion can be expressed as:

$$
\nabla\left(-\frac{\mu_{i}}{T}\right)=-\frac{\nabla_{T} \mu_{i}}{T}-h_{i} \nabla\left(\frac{1}{T}\right)
$$

By combining Eqs. (9) and (11), the component fluxes can be written as:

$$
\mathbf{J}_{\mathbf{i}}=-n_{i} L_{P}\left(\frac{\mathrm{V} \nabla P-M \mathbf{g}}{T}\right)+n_{i} \sum_{j} L_{i j}\left(\left(\frac{n_{j}}{n_{i}} h_{i}^{*}-h_{j}\right) \nabla\left(\frac{1}{T}\right)-\frac{\nabla_{T} \mu_{j}}{T}+\frac{M_{j} \mathbf{g}}{T}\right)
$$

The cross phenomelogical diffusion terms $L_{i j}$ are very difficult to get and can be considered to be small compared to the diagonal terms. This is of course not true for Fickian cross diffusion terms, even if they are usually small compared to the diagonal terms. The impact of chemical potential derivative matrix can even become singular at the critical point. Therefore, we consider: $L_{i j}=0$ if $i \neq j$ and $L_{i i}=L_{i}$ if $i=j$. In addition, if we use the following quantities:

$$
q_{i}=\frac{h_{i}-h_{i}^{*}}{T} \text { and } Q=\sum_{i} x_{i} \frac{h_{i}-h_{i}^{*}}{T}
$$

The final expressions of the molar fluxes become:

$$
\mathbf{J}_{\mathbf{i}}=-n_{i} L_{P}\left(\frac{\mathrm{V} \nabla P-M \mathbf{g}}{T}\right)+n_{i} \frac{L_{i}}{T}\left(q_{i} \nabla T-\nabla_{T} \mu_{i}+M_{i} \mathbf{g}\right)
$$




\section{Practical formulations}

\subsection{Pressure and chemical potential gradients}

\subsubsection{General case in an open system}

The purpose of this section is to derive pressure and chemical potential gradients within the framework provided in the previous section. To do so, we first sum the component fluxes divided by $L_{i}$ which gives

$$
\frac{M}{\rho} \sum_{i} \frac{\mathbf{J}_{\mathbf{i}}}{L_{i}}=-\frac{L_{P}}{L_{D}}\left(\frac{\mathrm{V} \nabla P-M \mathbf{g}}{T}\right)+\frac{Q}{T} \nabla \mathrm{T}-\frac{1}{T} \sum_{i} x_{i}\left(\nabla_{T} \mu_{i}-M_{i} \mathbf{g}\right)
$$

where $x_{i}$ is the mole fraction of component $i$ and $L_{D}$, the average diffusion mobility, is given by:

$$
\frac{1}{L_{D}}=\sum_{i} \frac{x_{i}}{L_{i}}
$$

Applying the Gibbs-Duhem relationship [7] in the thermal field and the gravity field yields:

$$
\sum_{i} x_{i}\left(\nabla_{T} \mu_{i}-M_{i} \mathbf{g}\right)=\sum_{i}\left(\nabla_{T} \mu_{i}-M_{i} \mathbf{g}\right)+S \nabla T=V \nabla P-M \mathbf{g}
$$

Then, it is possible to deduce the pressure gradient:

$$
\nabla P-\rho \mathbf{g}=\frac{\frac{\rho}{M} Q \nabla T-T \sum_{i} \frac{\mathbf{J}_{\mathbf{i}}}{L_{i}}}{\frac{L_{P}}{L_{D}}+1}
$$

and using Eq.(14) and Eq.(18) we obtain the chemical potential gradient at constant temperature:

$$
\nabla_{T} \mu_{i}-M_{i} \mathbf{g}=\left(q_{i}-\frac{L_{p}}{L_{i}}\left(\frac{Q}{\frac{L_{P}}{L_{D}}+1}\right)\right) \nabla T-\frac{M}{\rho} T\left(\frac{\mathbf{J}_{\mathbf{i}}}{x_{i} L_{i}}-\frac{\sum_{j} \frac{\mathbf{J}_{\mathbf{j}}}{L_{i}}}{L_{i}\left(\frac{L_{P}}{L_{D}}+1\right)}\right)
$$

It should be emphasized that, in our approach, the link between thermodynamic properties and transport properties is build-in. The stationary state is a balance between the various forces weighted by the mobility of each molecule.

There are some interesting features in Eq. (19):

- The separation in a thermal field is sensitive to the relative diffusion coefficients at stationary state. In porous media, the separation is sensitive to the permeability (through $L_{P}$ coefficient) when the overall mobility $L_{P}$ is similar to the average diffusive mobility $L_{D}$. 
- The magnitude of the separation depends on $q_{i}$ quantities: difference between the enthalpy of the component in the bulk flow and the enthalpy of the same component moving independently through a diffusion process.

- External fluxes impact the compositional distribution, as expected from fluxes, cf. eq. (12). External fluxes values that just cancel the thermodiffusion are given by $\mathbf{J}_{\mathbf{i}}=\frac{\rho}{M} x_{i} L_{i} \frac{q_{i}}{T} \nabla T$

\subsubsection{Closed system}

In a closed system, the previous relations reduce to:

$$
\begin{gathered}
\nabla_{T} \mu_{i}-M_{i} \mathbf{g}=\left(q_{i}-\frac{L_{p}}{L_{i}}\left(\frac{Q}{\frac{L_{p}}{L_{D}}+1}\right)\right) \nabla T \\
\nabla P-\rho \mathbf{g}=\frac{\rho}{M}\left(\frac{Q}{\frac{L_{p}}{L_{D}}+1}\right) \nabla T
\end{gathered}
$$

As expected, summation of Eq. (20) verifies Gibbs-Duhem [7] relation:

$$
\sum_{i} x_{i}\left(\nabla_{T} \mu_{i}-M_{i} \mathbf{g}\right)=\frac{\mathrm{Q}}{\frac{L_{p}}{L_{D}}+1} \nabla T=\frac{M}{\rho}(\nabla P-\rho \mathbf{g})=V \nabla P-M \mathrm{~g}
$$

\section{Permeable porous media case}

In case of a high overall mobility $\left(L_{P}>>L_{D}\right)$, Eq.(20) reduce to:

$$
\nabla_{T} \mu_{i}-M_{i} \mathbf{g}=\left(q_{i}-\frac{L_{D}}{L_{i}} Q\right) \nabla T
$$

and according to Eq.(21) the pressure gradient becomes hydrostatic: $\nabla P=\rho \mathbf{g}$. This is true only if $L_{P}>>L_{D}$, we will see in $\S 3.3$ that this is true for most of the petroleum reservoirs except for the unconventional play (Oil and Gas shale). Practical expressions for $L_{P}$ and $L_{D}$ are given in \$3.3. Thus, in permeable porous media, the separation results from a balance between two forces. The thermal force pushes the molecules but the diffusion process holds it back.

\section{Low permeability porous media case}

In case of low overall mobility (tight porous media) Eq.(20) reduce to:

$$
\begin{aligned}
& \nabla_{T} \mu_{i}-M_{i} \mathbf{g}=q_{i} \nabla T \\
& \nabla P-\rho \mathbf{g}=\frac{\rho}{M} Q \nabla T
\end{aligned}
$$


Thus, in low permeability porous media, the thermal gradient induces the highest possible pressure gradient. Interestingly, without gravity, the pressure gradient becomes:

$$
\nabla P=\frac{Q}{V} \nabla T
$$

Applied to a pure compound this just means that $\nabla \mu=0$ and it leads to the Clapeyron equation [10] along an interface if $Q=S_{\text {res. }}$ (molar residual entropy). It is a strong indication that $q_{i}$ should be related to the molar residual entropy of component " $i$ " in a mixture. We will come back on that important point latter.

\subsubsection{Aerial thermal gradient}

In the case of a liquid vapour interface subjected to an aerial thermal gradient and a gravity, see

Fig 1, which is sometimes found in large fields, it is possible to derive the tilt angle from Eq. 18 by applying this equation in both phase along the interface.

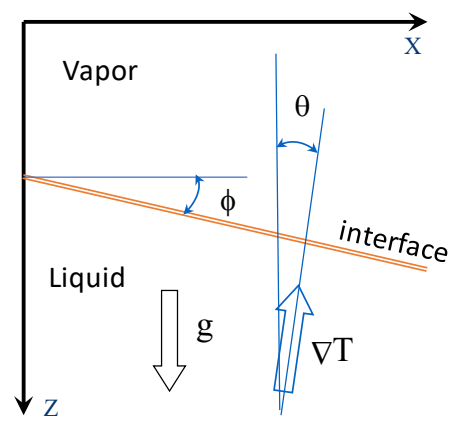

Fig.1: Interface subjected to gravity and thermal gradient

For a small angle:

$$
\sin \varphi=\frac{\delta}{1+\delta} \sin \theta \text { with } \delta=\frac{1}{\left(\rho_{l}-\rho_{v}\right) \mathrm{g}}\left(\frac{\frac{Q_{v}}{V_{v}}}{1+\frac{L_{p v}}{L_{D v}}}-\frac{\frac{Q_{l}}{V_{l}}}{1+\frac{L_{p l}}{L_{D l}}}\right) \frac{d T}{d x}
$$

As expected, for high permeability $\left(L_{p} \gg L_{D}\right)$, the tilt angle is nil. For low permeability $\left(L_{p} \ll L_{D}\right), \delta$ reduced to:

$$
\delta=\frac{\left(\frac{Q_{v}}{V_{v}}-\frac{Q_{l}}{V_{l}}\right)}{\left(\rho_{l}-\rho_{v}\right) g} \frac{d T}{d x}
$$




\subsection{Link with usual thermodynamic properties}

The difference between the value $K$ of any thermodynamic function of fluid at $P$ and $T$, and its value $K^{\circ}$ at the same temperature calculated for an ideal gas behaviour at reference pressure $P^{\circ}$, or reference volume $V^{\circ}$, is denoted as a departure from the ideal behaviour, a configurational property, or a residual part of the thermodynamic function, $\Delta K^{o}$. The link between the two configurational properties is [10]:

$$
\Delta K_{T, V}^{o}=\Delta K_{T, P}^{o}+\int_{\frac{R T}{V}}^{P}\left(\frac{\partial K_{T, P}^{o}}{\partial P}\right)_{T, n} d P
$$

Our approach is based on material and enthalpy balance for an elementary volume. Therefore, we use $V$ and $T$ reference conditions to link thermodynamic and transport properties in the following. It should be noticed that ideal enthalpy using $P$ and $T$ or $V$ and $T$ reference conditions are the same but ideal entropy and ideal chemical potential are different.

The chemical potential of a component can be replaced by its fugacity coefficient $\varphi_{i}$ :

$$
\mu_{i}=R T \log \varphi_{i}+\mu_{i(T, P)}^{o}=R T \log \varphi_{i}+\mu_{i(T, V)}^{o}+R T \log Z
$$

where $\mathrm{R}$ is the gas constant and $Z=P V / R T$ is the compressibility factor. It is then possible to express the residual parts of the chemical potential, enthalpy and entropy as:

$$
\begin{gathered}
\mu_{i}^{r e s}=R T \log \varphi_{i}+R T \log Z, \\
h_{i}^{r e s}=R T^{2} \frac{\partial \log \varphi_{i}}{\partial T}, \\
s_{i}^{r e s}=-R \log \varphi_{i}-R T \frac{\partial \log \varphi_{i}}{\partial T}-R \log Z
\end{gathered}
$$

It is observed that the diffusive part of the flux of a component through the elementary volume is driven by its chemical potential gradient and temperature gradient, see Eq. (6). We concluded that the enthalpy of the component in the bulk flow $h_{i}$ is different from the enthalpy of the same component moving alone independently through a diffusion process $h_{i}^{*}$. However, the chemical potential is the same for the diffusing component at the boundary in and out the elementary volume, i.e. $\mu_{i}^{*}=\mu_{i}$. This implies that:

$$
q_{i}=\frac{h_{i}-h_{i}^{*}}{T}=\frac{\mu_{i}-\mu_{i}^{*}}{T}+s_{i}-s_{i}^{*}=s_{i}-s_{i}^{*}
$$

At this point, we assume that the entropy of the component " $i$ ' alone diffusing in and out the elementary volume is its ideal entropy at reference $T$ and $V$ conditions. Such a clear link between thermodiffusion and residual entropy is strongly supported by recent results obtained from molecular simulations of isotopic mixtures [11] and is consistent with what known for other 
transport properties [12-13]. It is also compulsory to be consistent with the Clapeyron equation along a vapour/liquid interface.

But it remains an assumption until we could make a clear evaluation of the heat transfer through molecular diffusion.

It is then possible to deduce that:

$$
\begin{gathered}
q_{i}=s_{i}-s_{i}^{*}=s_{i}^{r e s}=-R \log \varphi_{i}-R T \frac{\partial \log \varphi_{i}}{\partial T}-R \log Z \\
Q=S^{r e s}=-R \log Z-R \sum_{i} x_{i}\left(\log \varphi_{i}+T \frac{\partial \log \varphi_{i}}{\partial T}\right)
\end{gathered}
$$

and, at a consequence, $h_{i}^{*}=h_{i}^{o}+\mu_{i}^{r e s}$, where the enthalpy of the component moving alone through a diffusion process $h_{i}^{*}$ is the ideal enthalpy of the component plus the residual part of the chemical potential.

With an explicit gravitational potential the chemical potential gradient of molecule " $i$ " is given by Eq. 19:

$$
\nabla_{T} \mu_{i}=M_{i} \mathbf{g}+\left(q_{i}-\frac{\frac{L_{p}}{L_{i}} Q}{\frac{L_{p}}{L_{D}}+1}\right) \nabla T-\frac{M}{\rho} \frac{T}{L_{i}}\left(\frac{\mathbf{J}_{\mathbf{i}}}{x_{i}}-\frac{\sum_{k} \frac{L_{p}}{L_{k}} \mathbf{J}_{\mathbf{k}}}{\frac{L_{p}}{L_{D}}+1}\right)
$$

which reduces to, without external fluxes (closed system) in a permeable system:

$$
\nabla_{T} \mu_{i}=M_{i} \mathbf{g}+\left(q_{i}-\frac{L_{D}}{L_{i}} Q\right) \nabla T
$$

The chemical potential gradient can then be replaced by compositional gradients:

$$
\nabla_{T} \mu_{i}=R T \sum_{j}\left(\frac{\delta_{i j}}{x_{j}}+\frac{\partial \log \varphi_{i}}{\partial x_{j}}\right) \nabla x_{j}+v_{i} \nabla P
$$

And, starting from a reference point at which the fluid properties are known, the new compositions are obtained by solving a set of linear equations.

\subsection{Link with usual transport properties}

The phenomenological coefficients defined so far, $L_{i}, L_{p}, L_{q j}$ are not practical quantities. Thus, in the following we link these coefficients with more usual quantities. The phenomenological advection coefficient is simply deduced from the fact that the advective fluid velocity without chemical and thermal forces is described by a simple Darcy's law [6]:

$$
\mathbf{U}=-\frac{k}{\eta}(\nabla P-\rho \mathbf{g})
$$

where, $k$ is the permeability of the porous medium and $\eta$ the viscosity of the fluid. From Eq. (3), therefore, it is possible to deduce that : 


$$
L_{P}=\frac{\rho}{M} T \frac{k}{\eta}=\frac{P}{Z R} \frac{k}{\eta}
$$

The phenomenological diffusion coefficient $L_{i}$ can be linked to the usual effective diffusion coefficient $D_{i}$, only when dealing with ideal mixtures: $L_{i}=D_{i} / R$.

The average diffusion mobility $L_{D}$ is linked to usual effective diffusion coefficient $D_{i}$ of the components [6], therefore:

$$
\frac{L_{i}}{L_{D}}=\sum_{k} \frac{x_{k}}{L_{k}} L_{i}=D_{i} \sum_{i} \frac{x_{k}}{D_{k}}
$$

In the general case, using eq. (37), we have :

$$
L_{i}\left[\left(1+x_{i} \frac{\partial \log \varphi_{i}}{\partial x_{i}}\right) \nabla x_{i}+x_{i} \sum_{k \neq i} \frac{\partial \log \varphi_{i}}{\partial x_{k}} \nabla x_{k}\right] \equiv \frac{D_{i}}{R} \nabla x_{i}
$$

And the evaluation of the phenomenological diffusion coefficient $L_{i}$ cannot be made straightforwardly from usual diffusion coefficients. However, for a dilute component in a solvent mixture, far from critical conditions, we can approximate our mobility coefficients by :

$$
L_{i}=\frac{D_{i}}{R\left(1+x_{i} \frac{\partial \log \varphi_{i}}{\partial x_{i}}\right)}
$$

In dense fluids, the effective infinite dilution diffusion coefficient of a species is roughly inversely proportional to the section of the molecule, i.e. a volume at an exponent $\tau$ of about $2 / 3$. For practical application with an equation of state, as will be seen in the following, we assume that $D_{i}$ is proportional to $b_{i}{ }^{-\tau}$, where $b_{i}$ is the covolume of the studied component [10] defined as $b_{i} \propto T c_{i} / P c_{i}$ where $T c_{i}$ and $P c_{i}$ are the critical temperature and the critical pressure of component $i$ respectively. With this formulation and assuming that all components are diluted, we can deduce that:

$$
\frac{L_{i}}{L_{D}}=L_{i} \sum_{k} \frac{x_{k}}{L_{k}}=\frac{\left(\frac{T c_{i}}{P c_{i}}\right)^{-\tau}}{\left(1+x_{i} \frac{\partial \log \varphi_{i}}{\partial x_{i}}\right)} \sum_{k} x_{k} \frac{\left(1+x_{k} \frac{\partial \log \varphi_{k}}{\partial x_{k}}\right)}{\left(\frac{T c_{k}}{P c_{k}}\right)^{-\tau}}
$$

We use $\left(T c_{i} / P c_{i}\right)^{-\tau}$ for practical reasons, the right quantities are the square of molecular size. In addition, since $L_{P}$ and $L_{D}$ are both inversely proportional to the fluid viscosity, it is interesting to notice that the $L_{P} / L_{D}$ ratio in equations (35) and (36) is independent of the fluid viscosity. In dense fluid, this ration depends mainly on the ratio between the size of the molecules and the size of the pore throat.

For typical reservoir fluid composition and typical reservoir conditions, the ratio $L_{P} / L_{D}$ becomes close to unity when the permeability is in the range 10 to 100 nanoDarcy which 
corresponds to the permeability of shale. In the case of usual reservoir permeability this ratio is very large and equation (36) can be applied to get the compositional gradient.

\subsection{Practical resolution algorithm}

For practical applications, one can use an equation of state (EoS) model, in that work a classical Peng-Robinson EoS with Peneloux volume translation [10], to compute all quantities [5, 14]. The simplest formulation is to calculate the composition at a given depth, $(Z)$, and temperature, as a function of a reference point "ref" where all properties are known. Starting from Eq. (36) we get:

$$
\frac{x_{i}}{x_{i}^{r e f}}=\frac{P^{r e f}}{P} \frac{\varphi_{i}^{r e f}}{\varphi_{i}} e^{\left(M_{i} \mathbf{g}+\left(q_{i}-\frac{L_{D}}{L_{i}} Q\right) \nabla T\right) \frac{\left(z-z^{r e f}\right)}{R T}}
$$

where fugacity coefficients have to be evaluated at the same temperature. If the gravity forces are not balanced, the new pressure $P$ is equal to the reference pressure plus the weight of the fluid column. Thus, the contribution of the pressure to the fugacity ratio can be included in the gravity term:

$$
\frac{x_{i}}{x_{i}^{r e f}}=\frac{\varphi_{i}^{r e f}}{\varphi_{i}} e^{\left(\left(M_{i}-\rho v_{i}\right) \mathbf{g}+\left(q_{i}-\frac{L_{D}}{L_{i}} Q\right) \nabla T\right) \frac{\left(z-z^{r e f}\right)}{R T}}
$$

Where

$$
v_{i}=\frac{R T}{P}+R T \frac{\partial \log \varphi_{i}}{\partial P}
$$

In this case, the fugacity at the point of interest must be calculated at the same pressure than the reference point.

This set of equations clearly shows the competition between gravity and thermodiffusion forces. A perfect balance is obtained if:

$$
\left(M_{i}-\rho v_{i}\right) \mathbf{g}+\left(q_{i}-\frac{L_{D}}{L_{i}} Q\right) \nabla T=0
$$

And external fluxes values that just cancel the thermodiffusion are given by:

$$
\mathbf{J}_{\mathbf{i}}=\frac{\rho}{M} x_{i} L_{i} \frac{q_{i}}{T} \nabla T
$$

\section{Application and validation}


In this section, the proposed approach has been applied on a realistic reservoir fluid in reservoir conditions to determine the influence of thermodiffusion on species vertical distribution. More precisely, a fluid column of $450 \mathrm{~m}$ height (located between $-5325 \mathrm{~m}$ and $-5775 \mathrm{~m}$ ) subjected to a geothermal gradient of $0.029 \mathrm{~K} / \mathrm{m}$ and gravity has been modeled. The temperature and the pressure at $-5525 \mathrm{~m}$ are $87.5^{\circ} \mathrm{C}$ and 641 bar, respectively.

\subsection{Parameterisation of the studied system}

It is worth mentioning that validation on a real field case is almost impossible because of the uncertainty on fluid samples. There are also various factors that may impact the compositional gradient [6] and it is rather difficult to be sure that the stationary state is reached. Therefore, to apply and verify the proposed approach we used a realistic fluid composition but, instead of using real field data, the influence of gravity and thermal gradient were evaluated through NonEquilibrium Molecular Dynamics (NEMD) simulations based on the methodology described in refs. $[14,15]$.

Furthermore, modern analytical technics give us a huge number of components and it is not possible to use a full fluid description of reservoir fluid in NEMD simulation. Thus, we used a thermodynamic lumping technique, to reduce the number of components of the studied oil to a manageable value ( 8 pseudo-components in this case as shown in Table 1). More precisely, lumping was achieved a standard approach based on volatility under engineering constraints (groups correspond to boiling points cuts). The small amount of nitrogen was lumped with methane (N2C1) and the heavy ends $\mathrm{C} 20+$ was divided in two cuts (CN1 and CN2).

The properties of the pseudo-components (critical properties, $T_{c}$ and $P_{c}$, and acentric factors, $\omega)$ were deduced from the constraint of iso-chemical potential at initial reservoir conditions because EOS parameters of the pseudo component must be the same than calculated values from EOS mixing rules based on initial reservoir fluid compositions. The usual properties of the pseudo-components (critical parameters and acentric factors) were deduced from the calculated EOS parameters. And volume translation parameters $C_{i}$ were obtained by averaging the pure component values.

\begin{tabular}{crrrrrr}
\hline $\begin{array}{c}\text { Pseudo- } \\
\text { components }\end{array}$ & $\begin{array}{c}\text { Mole \% } \\
\text { @5525m }\end{array}$ & $M\left(\mathrm{~g} / \mathrm{mol}^{-1}\right)$ & $T_{c}(\mathrm{~K})$ & $P_{c}$ (bar) & $\omega$ & $C_{i}$ \\
\hline N2C1 & 36.273 & 16.12 & 190.26 & 46.33 & 0.0117 & -0.220 \\
CO2 & 37.262 & 44.01 & 304.10 & 73.70 & 0.2389 & -0.030 \\
\hline
\end{tabular}




\begin{tabular}{ccccccc}
\hline C2 & 4.795 & 30.07 & 305.43 & 48.84 & 0.0986 & -0.070 \\
C3C4 & 5.373 & 49.52 & 388.85 & 40.40 & 0.1653 & -0.010 \\
C5C10 & 6.061 & 99.29 & 572.15 & 30.20 & 0.2924 & 0.020 \\
C11C19 & 4.948 & 196.19 & 728.15 & 20.60 & 0.4746 & 0.025 \\
CN1 & 2.849 & 331.82 & 883.15 & 15.20 & 0.7300 & 0.030 \\
CN2 & 2.439 & 569.31 & 1173.15 & 13.50 & 0.9790 & 0.035 \\
\hline
\end{tabular}

Table 1 : Thermodynamic properties of pseudo-components used to describe the oil.

The transcription of the properties of the pseudo-component into molecular models used in molecular dynamics has been achieved using a top-down strategy based on the corresponding states, similarly to what done in ref. [16]. More precisely, we have used the Lennard-Jones chains model to represent the pseudo-component in which the molecular parameters $(\sigma$. sphere diameter, $\varepsilon$ : potential depth and $N$ : number of segments) were adjusted so as to yield exactly the critical temperature and the critical pressure, except for $\mathrm{CO}_{2}$. All molecular parameters so defined are provided in Table 2. For $\mathrm{CO}_{2}$ we have used the force field developed in ref. [17]. Classical Lorentz-Berthelot combining rules were used to define the cross molecular parameters. This coarse grained molecular model, even if perfect, is known to describe reasonably well thermodiffusion and PVT properties of hydrocarbons mixtures $[5,14]$.

\begin{tabular}{cccc}
\hline $\begin{array}{c}\text { Pseudo- } \\
\text { components }\end{array}$ & $N$ & $\varepsilon\left(\mathrm{j} . \mathrm{mol}^{-1}\right)$ & $\sigma(\AA)$ \\
\hline N2C1 & 1 & 1209 & 3.697 \\
C2 & 2 & 1438 & 3.289 \\
C3C4 & 2 & 1831 & 3.760 \\
C5C10 & 3 & 2277 & 4.095 \\
C11C19 & 5 & 2501 & 4.149 \\
CN1 & 8 & 2798 & 4.222 \\
CN2 & 10 & 3459 & 4.468 \\
\hline
\end{tabular}

Table 2 : Molecular parameters of pseudo-components used to describe the oil

The last parameter to define is the exponent $\tau$ appearing in the mobility ratio of Eq. (43): 


$$
\frac{L_{i}}{L_{D}}=L_{i} \sum_{k} \frac{x_{k}}{L_{k}}=\frac{\left(\frac{T c_{i}}{P c_{i}}\right)^{-\tau}}{\left(1+x_{i} \frac{\partial \log \varphi_{i}}{\partial x_{i}}\right)} \sum_{i} \frac{x_{k}}{\left(\frac{T c_{k}}{P c_{k}}\right)^{-\tau}}\left(1+x_{k} \frac{\partial \log \varphi_{k}}{\partial x_{k}}\right)
$$

As an initial value we have chosen to take $\tau=0.6$ as done in the correlation of Wilke and Chang which is known to apply well on the hydrocarbon homologue series [10]. It should be recalled that the applicability of this relation is based on the fact that most of the component can be considered as "diluted" in the reservoir fluid. It is obviously the case of the components of the liquid fraction but it is not the case of some light components $\left(\mathrm{CH}_{4}\right.$ and $\mathrm{CO}_{2}$ in our example). We assume that such an approximation remains valid for these small molecules in the reservoir fluid.

\subsection{Results}

As mentioned previously, a fluid column of $450 \mathrm{~m}$ height was simulated by NEMD subject to gravity with, and without, a realistic thermal gradient of $0.029 \mathrm{~K} / \mathrm{m}$ to build the reference solution used to validate the thermodynamic model developed in this work. NEMD compositional profiles of some of the pseudo component are shown in Figs. 1 and 2. Then, computations have been achieved using the proposed thermodynamic model. The composition at depth $-5525 \mathrm{~m}$ was used as a reference for EOS modelling, EOS calculations make use of a reference depth composition, NEMD simulation use an average composition. For sake of comparison we use the composition at $5525 \mathrm{~m}$ obtained from anisothermal NEMD simulation as the reference composition for EOS calculation. Since the average composition depends on the profile, it is not possible to get the same reference composition for the different cases.
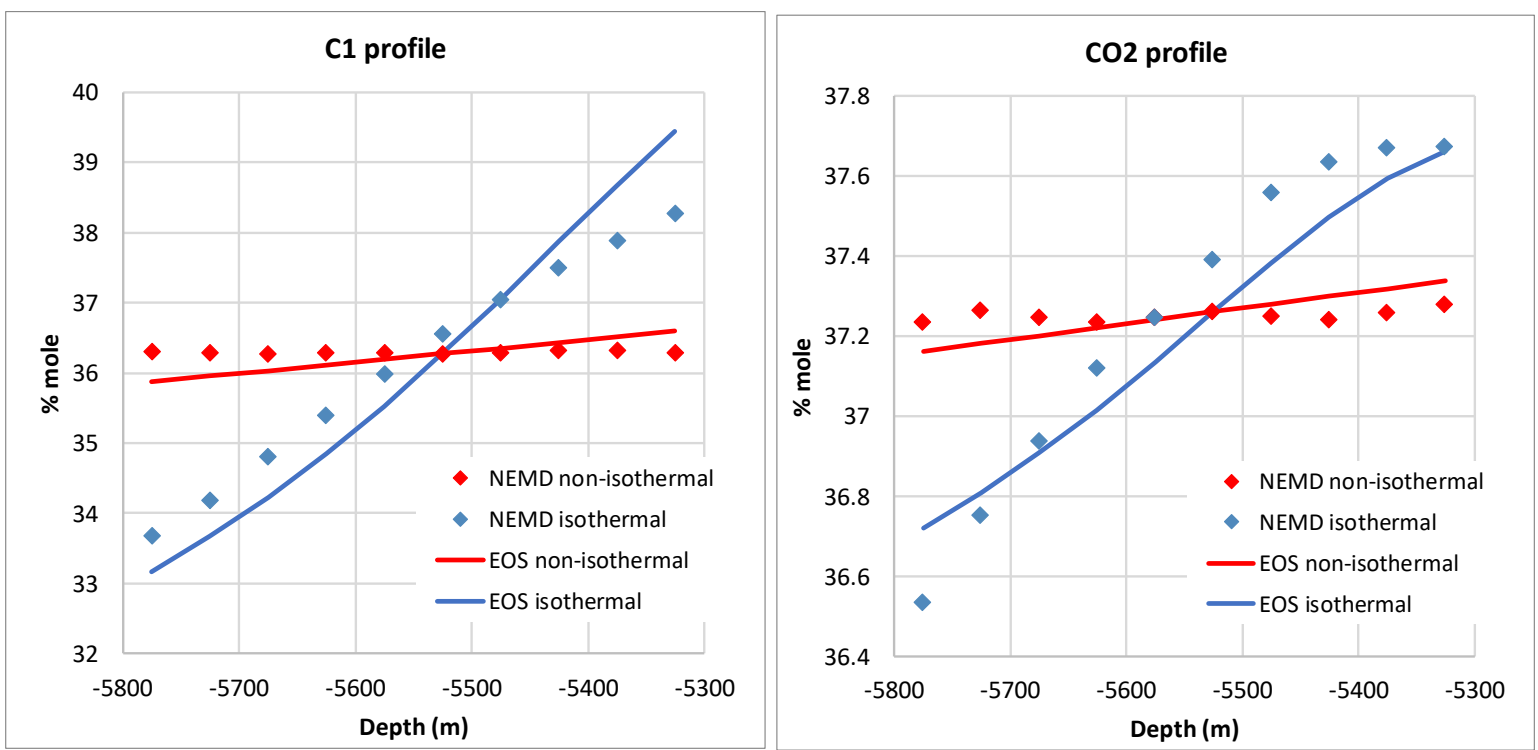
Figure 2: Compositional profile of the two lightest compounds of the oil.
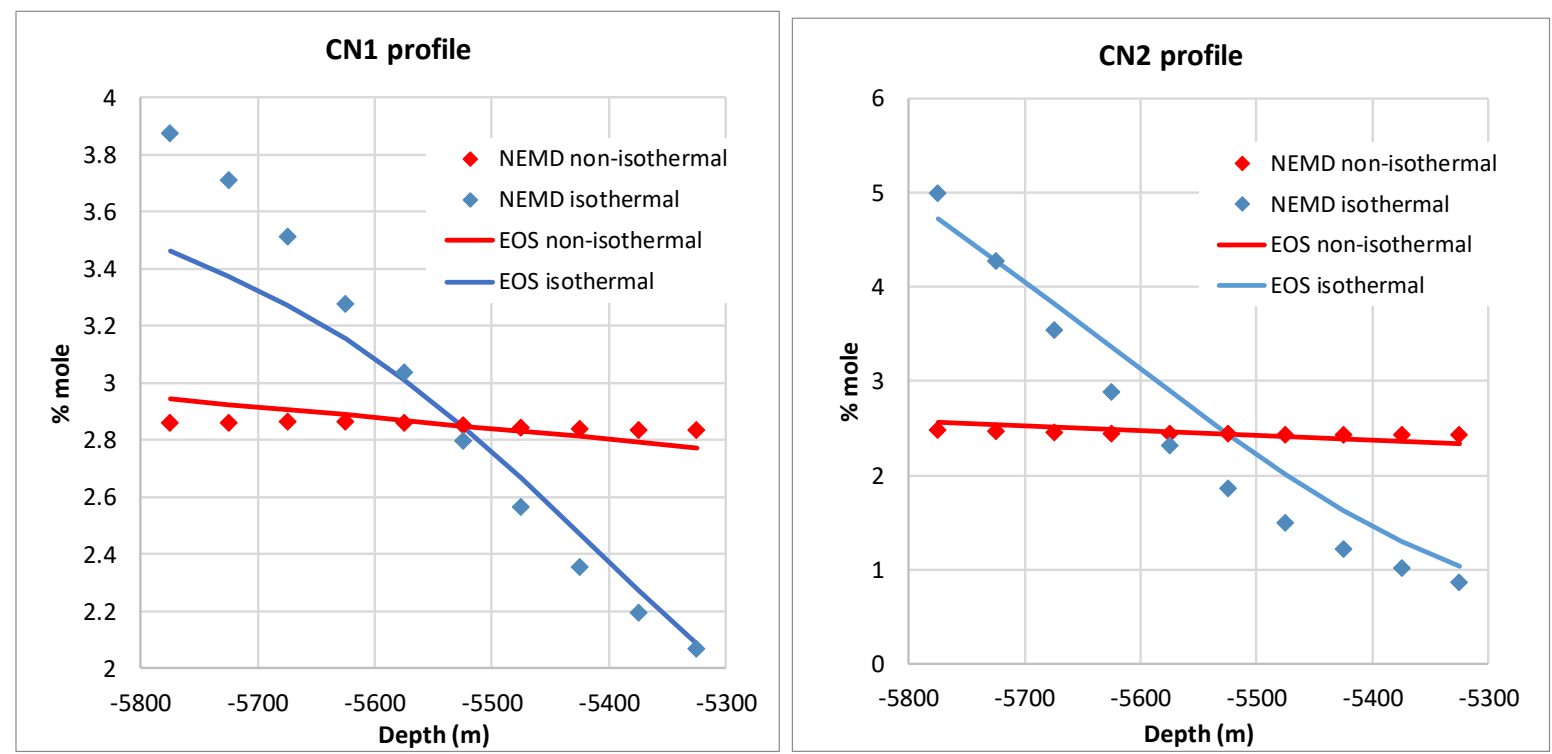

Figure 3: Compositional profile of the two heaviest compounds of the oil.

First, it is interesting to notice, see Figs. 2 and 3, that this realistic thermal gradient leads to an almost perfect compensation of the gravity force by the thermodiffusion force. Even, if this behavior has been noticed for other systems, such as acid gas [5] or synthetic oil [14], such a behavior is particularly striking for the heavy cuts (CN1 and CN2) which are highly segregated when gravity alone is applied.

Second, Figures 2 and 3 show a qualitative agreement between results from NEMD simulations and those obtained from the proposed thermodynamic models with or without thermal gradient. Thus, as the results obtained by the two approaches without gravity are similar, this indicates that the thermodynamic model proposed in this work, despites its intrinsic assumptions, captures well the influence of thermodiffusion on the compositional profiles without any fitting parameters. However, it should be pointed out that the sensitivity to exponent $\tau$ is large as shown in Fig. 4 and that a better fit could be obtained for $\tau=0.59$. 


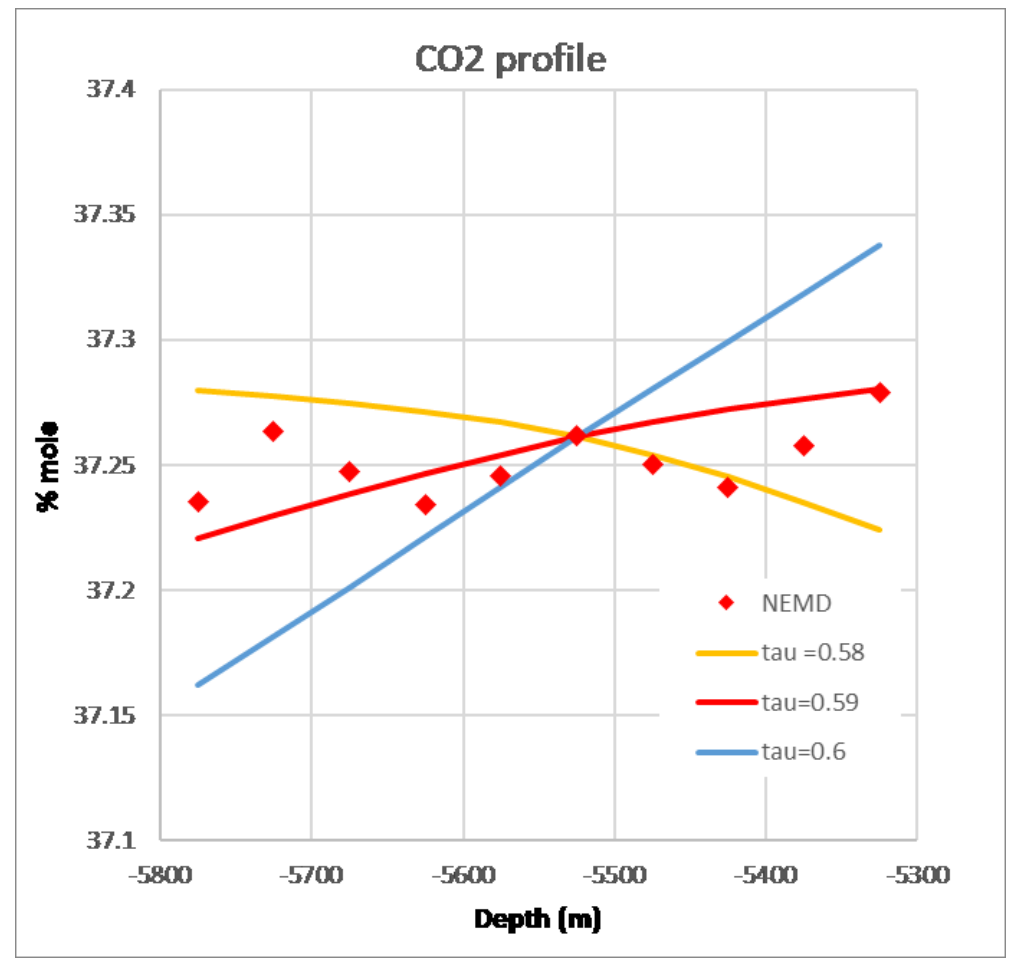

Figure 4: Compositional profile of $\mathrm{CO}_{2}$, sensitivity tests on $\tau$

From this application we can deduced that our approach is good enough for practical application in the petroleum industry. But it may be due to the large number of components in the reservoir fluids and it could be necessary for other mixtures to account for cross phenomenological coefficients in Eq. (2). One of the key figures is that the molecular diffusive mobility appears only as divided by the average diffusive mobility (Eq.(43)). Therefore the evaluation of this ratio do not require information on the $\mathrm{P}, \mathrm{T}, \rho$ dependency of these coefficients. We just need to know how these coefficients vary with the properties of the molecule itself. The other part of the thermal force is a simple thermodynamic property and accurate enough when the EOS model have been tuned on a large set of experimental data.

\section{Conclusions}

Thanks to an irreversible thermodynamic approach, we developed expressions providing the chemical potential gradients and the pressure gradient induced by the geo-thermal field in a petroleum reservoir. In the final expressions, the chemical potential gradients are proportional to the temperature gradient, and the proportionality coefficients are molar residual entropy of 
each component balanced by the average residual entropy multiplied by the relative diffusion mobility of the components.

The obtained relations imply that the species relative separation in a thermal field is sensitive to the relative diffusion coefficients at stationary state. In porous media, the separation is sensitive to the permeability when the overall mobility is similar to diffusive mobility. In addition, the magnitude of the separation depends on the residual entropy of the species and the separation is not simply balanced by the average residual entropy. The balance is modified by the relative diffusion mobility of the components. Another interesting feature of the proposed relations is that in low permeability porous media, the thermal gradient induces a pressure gradient proportional to the fluid residual entropy.

Combined with an equation of state and a classical correlation for transport properties, the proposed relations do not need fitting parameters to model the impact of the thermal gradient on pressure and compositional gradients. As a test, the proposed approach has been applied on an eight pseudo-components reservoir fluid subjected to a geothermal gradient. First results in terms of compositional profiles versus depth have shown to be in good agreement with nonequilibrium molecular dynamics simulation results at the stationary state.

\section{Acknowledgements}

The authors would like to thank all the colleagues who have contributed to this study and especially Alexander Shapiro from DTU for fruitful discussions. We are also very grateful to Total S.A. for its support during many years.

\section{References}

[1] W. Köhler and K. I. Morozov, J. Non-Equilib. Thermodyn. 41, 151 (2016).

[2] A. Firoozabadi, Thermodynamics and Applications in Hydrocarbon Energy Production, Mac Graw Hill (2015).

[3] R. O. Esposito, P. H. R. Alijó, J. A. Scilipoti, F. W. Tavares, Compositional Grading in Oil and Gas Reservoir, $1^{\text {st }}$ Edtion, Gulf Professional Publishing (2017).

[4] K. Ghorayeb, A. Firoozabadi, T. Anraku, SPE Journal 8, 114 (2003).

[5] G. Galliero, H. Bataller, F. Croccolo, R. Vermorel, P. A. Artola, B. Rousseau, V. Vesovic, M. Bou-Ali, J. M. O. de Zarate, S. Xu, K. Zhang, F. Montel, Microgravity Sci. Technol. 28, 79 (2016).

[6] F. Montel, J. Bickert, A. Lagisquet, G. Galliéro, J. Petrol. Sci. Eng. 58, 391 (2007).

[7] S. R. De Groot, P. Mazur, Nonequilibrium Thermodynamics, Dover, New York (1984). 
[8] R. Haase, Thermodynamics of irreversible processes, Reading, Mass., Addison-Wesley (1969).

[9] K. S. Pedersen and N. Lindeloff, Simulations of Compositional Gradients in Hydrocarbon Reservoirs Under the Influence of a Temperature Gradient, SPE Annual Technical Conference and Exhibition, (2003).

[10] B. E. Poling, J. M. Prausnitz, J. P. O’Connell, The properties of Gases and Liquids, $5^{\text {th }}$ Edition, McGraw-Hill (2001).

[11] H. Hoang, G. Galliero, R. Vermorel, F. Montel, in preparation.

[12] Y. Rosenfeld, J. Phys. Condens. Matter 11, 5415 (1999).

[13] G. Galliero, C. Boned, J. Fernandez, J. Chem. Phys. 134, 064505 (2011).

[14] M. Touzet, G. Galliero, V. Lazzeri, M. Z. Saghir, F. Montel, J. C. Legros, CR Mécanique 339, 318 (2011).

[15] G. Galliero, F. Montel, Phys. Rev. E 79, 6413 (2009).

[16] H. Hoang, S. Delage-Santacreu, G. Galliero, Ind. Eng. Chem. Res. 56, 9213 (2017).

[17] G. Galliero, C. Nieto-Draghi, C. Boned, J. B. Avalos, A. D. Mackie, A. Baylaucq, F. Montel, Ind. Eng. Chem. Res. 46, 5238 (2007). 